the eye, but nevertheless in a manner which will soon relieve the patient, and which at once assures the surgeon of his complete success. The slight but very gradual increase in diameter which the lower end of the instrument exhibits, suffices also to dilate the stricture somewhat; and a No.1 catheter may generally be made to follow, on the withdrawal of the first instrument, a short time after its introduction. If doubt as to the practicability of this be entertained, a fine gum elastic tube may be passed into the bladder by first removing the handle and stylet, and screwing in its place a long steel rod, over which the tube may glide. No doubt that the safest and most satisfactory practice is to permit a small catheter to remain in the urethra a few hours until it can be replaced by a larger, and for this purpose it is convenient to employ a small bent tube, containing a stopcock, and adapted to the catheter, so that the patient can easily command the outflow of his urine, and at all events be secured from the unpleasant results which sometimes occur, when, a mere plus of wood being placed in its orifice, the urine dribbles backwards along the shaft of the catheter upon the patient's person. An entire set of catheters may be supplied with a single tube which fits every one of them as in a set, which I have long employed for the purpose, and which may be seen here.

$$
\text { [To be continued.] }
$$

Wimpole Street, Cavendish Square, 1857.

\section{CASES OF STRANGULATED HERNIA.}

By Draper Mackinder, M.D., F.R.C.S.Edin., etc., Gainsborough.

Fortunately for suffering humanity, the introduction of chlo roform has rendered the surgeon's knife not only less formidable, but less frequently necessary ; and, perhaps, there is no class of our patients more deeply indebted to the friendly anæsthetic than the poor victims of hernia.

A rupture is an accident of frequent occurrence in this neighbourhood, often attributable, I think, in working people to the foolish custom of wearing tight inelastic belts and stays.

Several cases of strangulation have been under my care during the last few years, but I have only had occasion to operate in five, four of which recovered.

A short account of these, from the notes in my day-book, will not perhaps be unacceptable.

CASE I. The first case was that of a labouring man, aged 65 who had had a right inguinal hernia for twenty years. It had always been reducible, and had been supported by a truss until a few weeks previously, when he had been induced to leave the instrument off. After a hard day's work at the end of June, the gut came down, and was soon followed by the usual symptoms of pain, vomiting, etc. Strangulation had existed for twentyfour hours when I first saw him.

In consequence of the great tenderness over the hernial protrusion and the severity of the constitutional symptoms, after a short trial of the taxis under chloroform, I proceeded to operate by the old plan of opening the sac, which I found adherent. The man was kept well under the influence of opium for three days, and left his bed well in a fortnight.

Case II. The second case was a laundress, aged 45, a delicate woman of a spare habit of body, who had had a right femoral hernia for a long time, but had never attended to it. After lifting a heavy tub, she felt something give way, and was afterwards seized with pain, chilliness, and vomiting. I was not sent for until the strangulation had existed four days, when there were fæcal vomiting, great tympanitis, and jactitation. Reduction had been tried by a medical friend; and a short application of the taxis, with anrsthesia, not affording relief, I immediately cut down, opened the sac, divided the stricture, and returned the chocolate-coloured intestine.

The poor woman was kept under the influence of opium and ammonia for some days, and, with the assistance of good support, eventually did well.

CASE IIr. The third was a case of left femoral bernia, oc. curring in a charwoman, aged 40 . This woman had not previously observed a swelling, but felt something give way after jumping out of bed in a state of alarm, and using some exertion to open a window. When seen, she was cold, vomiting, and suffering great pain in the abdomen, which was tympanitic. The vomited material being stercoraceous, attention was at once directed to the usual hernial apertures, and a small knuckle of intestine detected in the left femoral region.

After the common remedies had failed to effect reduction,
I proceeded to operate, and divided the stricture without opening the sac. The gut was easily returned, and the woman was quite well in ten days.

CASE IV. A woman, Mrs. W., aged 48, of moderate circumstances, but immoderate size, weighing twenty-two stones, is the next on my list. This unfortunate patient had had an immense irreducible hernia for fourteen years, from which she had occasionally suffered so much, that on my first visit, Nov. 2nd, 1855, she declared her suffering to be wholly independent of the rupture. She was accordingly treated for colic; but the next day she had stercoraceous vomiting, and an aggravation of all the other symptoms. Fearing she would die, I advised her to avail herself of the poor chance that remained, and sub. mit to an operation; to which she then consented, and was immediately put under chloroform. The tumour was about the size of a child's head. The incision through the integuments was some eight inches long, and through a stratum of adipose tissue at least four inches thick. The intestine was found encircled by numerous bands of lymph, which were broken up, the gut replaced in its natural position, and the wound united by sutures, immense pieces of strapping, and several yards of bandages; for so enormously large was the pendulous abdomen, that the poor woman could only turn over by giving the body a complete swing. The sutures were soon torn out and useless. On the following day Mrs. W. appeared calm and comfortable, and the bowels had operated many times. On the third day, she was sitting upon the commode when I entered the room, and expressed herself well. The pain had subsided; but there was still occasional vomiting of a little fæcal matter up to the tenth day after the operation, when she had a sudden rigor, followed by pain in the right hypochondriac region, collapse, and death.

A post mortem was not allowed.

From the vomiting which followed the operation, and never entirely ceased till her death, though the bowels were acted upon more than twenty times, despite the repeated administration of opiates, it is but natural to infer that a portion of gut had been unrelieved of its strangulation.

This was certainly the most formidable operation for hernia that I ever witnessed, and my hopes for her recovery were never very sanguine. Indeed, from the all but universally fatal result of operations for umbilical hernia, nothing but a strong sense of duty would induce any one to use the knife in such cases.

CASE v. In the evening of September 13th, 1856, I was requested by my assistant, Mr. Henry Towle, to visit a man who had got a strangulated inguinal hernia, which he could not reduce. The patient, Geo. Smith, aged 29, a fair complexioned, stout, healthy sailor, who had been on shore a few days, gave the following history of his case. He was a volunteer during the disturbance in New Zealand in 1845 ; and, in consequence of the breaking of a hook of the scaling ladder which he was ascending at the block house of Kororareka, Bay of Islands, he fell a distance of fourteen feet astride some pailings, and injured his perinæum. Next day he perceived a small lump in the groin, and his testicles were inflamed, for which he was ordered a suspensory bandage. The lump in the groin continued when in the upright position; but no notice was taken until four years and a half afterwards, when an American surgeon ordered him to wear a truss. This he left off the day before the accident, considering himself cured.

On the morning the strangulation occurred, he ran a race for a dinner, won and ate it; and an hour and a half afterwards he felt very ill, went to bed, and discovered a large lump in his right groin. He complained of much pain at the pit of the stomach, had slight vomiting, and a quisk feeble pulse. There was a very tense oblong tumour extending from the symphysis pubis to within a couple of inches of the anterior superior spinous process of the ilium : in other words, an ellipsis with a conjugate diameter of about six inches. The taxis, with the ordinary concomitants, having failed, I at once, before the anæsthesia had passed off, cut down on the tumour, opened the sac, divided the stricture, and returned eighteen inches of congested intestine. A strong opiate was administered, the incision united by first intention, and the patient, to my surprise, was downstairs on the morning of the third day, and went to church on the seventh.

On a further examination, I found a considerable bulging of the right lower half of the abdominal wall, probably depending on a rupture of some of the fibres of the transverse or oblique muscles. For this I got the man to make himself a strong, broad, woollen belt to embrace the lower half of the abdomen, having a pad affixed thereto of sufficient size and 
thickness to exert the necessary pressure on the weakened region. With this belt and the ordinary truss Jack expressed himself equal to any amount of work, and on the tenth day after the operation he embarked, lion-hearted, for another journey through " the wide, wide, world."$$
\text { 第eritscope. }
$$

\section{MIDWIFERY AND DISEASES OF WOMEN.}

\section{BLISTERING THE CERVIX UTERI.}

Dr. Robert Johns has published in the Dublin Quarterly Journal of Medical Science for May 1857, some practical ob. servations on blistering the cervix uteri, as a remedial agent in the treatment of certain diseased conditions of that viscus. He says :-

"As it is familiarly known that leeches, when applied directly to the cervix uteri in inflammatory and other affections of that and nejghbouring organs, act far more beneficially than when employed externally, and at a greater distance from the seat of the disease (as first enjoined by M. Guilbert), - so, as blisters, when used externally, act sometimes salutarily in the cases now under consideration,-may we not reasonably expect that their direct application to the offending viscus will be still more likely to be productive of good. Actuated by this, as well as other reasons, I have made trial of their efficacy, and $I$ have not been disappointed in my expectations.

"The plan I adopt for blistering the cervix uteri is as follows :-The parts are first brought into view by means of a speculum, - I generally use Fergusson's, but with moderate care any other will answer as well. They are then to be well freed from any mucous or other discharge by a dry, soft sponge; sometimes the mucus is so adherent, particularly when exuding from the os, that it is necessary to damp the sponge for its removal; in all cases, however, the parts must be well dried; after which a concentrated solution of can. tharides in sulphuric ether, mixed with the ordinary solution of gutta percha in chloroform, in the proportion of two parts of the former solution to one of the latter, is to be rapidly rubbed on the cervix by means of a camel's-hair pencil two or three times, according to the effect produced, as indicated by the appearance of the part, or by the sensations of the patient

"My first essay was with vesicating collodion; but as it caused great pain, both during its application and for hours afterwards, only ceasing on the appearing of the watery discharge ; and as its operation was not sufficiently expeditious or efficacious, I had a strong solution of cantharides in chloroform, in which gutta percha was afterwards dissolved, prepared for me by Mr. Walsh, of Westland Row, which had the advantage of being painless during and after its use; but it was not so speedy in its action, nor so powerful in its effects as the collodion. Therefore, after consulting with several chemists in the city, I eventually applied to Mr. Williams (Dr. Butler's chemist), who, after having made several experiments, kindly presented me with a specimen of the preparation which I now use, and offer to the profession as being fully adequate to fulfil the indications required. It is frequently of advantage to keep open the blistered surface; and this is satisfactorily done by the weaker preparation in chloroform, which I denominate vesicating gutta percha, No. 2,-the ordinary one (vesicating gutta percha, No. 1) being rarely required for that purpose. For some days after the operation I direct vaginal injections of cold water to be used, and sometimes I wash over the parts with a weak preparation of nitrate of silver. At first I was in the habit of keeping open the blisters by some strong caustic, but I very soon learned that the weaker solution of cantharides answered the purpose better.

"During the appliance of this remedy the patient experiences a pricking, stinging pain, together with a sensation of heat, sometimes amounting to burning: it is very bearable, and ceases almost immediately; indeed, in some cases she will not tell you of it unless you ask her; in others a sweet, pungent taste and smell are experienced, or an ethereal odour is perceived on her breath by another. Very frequently, in fact generally, small vesications appear at the time, and a watery discharge sets in within half an hour afterwards (which has a scalding sensation whilst passing), sometimes even before the speculum has been withdrawn. This discharge, starching the linen, and in other respects similar to that from blisters externally formed, lasts commonly for three days, when to it suc. ceeds one of a slightly purulent nature, but not productive of pain. At this stage we shall find the epithelium thickened and raised, and coming off in patches, like bits of chewed paper; but, prior to it, vesications, like to those on the skin, are very plainly visible with their exudation. On more than one occasion I have seen a watery discharge produced by preparation No. 2, from a surface previously blistered, used to keep it open; and the same phenomenon has occurred on an ulcerated surface.

"Blistering the cervix uteri does not cause any unpleasant sensations towards the rectum, bladder, or neighbouring organs. I never saw strangury or such like affection thereby induced : on the contrary, I have employed it more than once when vesical irritation was present, which, so far from being increased, was completely removed by two applichtions.

"The average length of time for repeating this treatment is about six days, unless it be desirable to keep up the process; then, in that case, it would be about three days."

Dr. Johns relates a number of cases in illustration of his practice, of which we quote several.

"C CASE I. Mrs. D., aged 34, of a strumous habit, several years married, but never pregnant, was under treatment for chronic inflammation of the cervix uteri, with endocervicitis, which gave way to local bleeding by scarification, the application of pernitrate of mercury alternating with a solution of nitrate of copper to the canal of the cervix, with the internal use of alteratives, cathartics, and tonics; however, pain in the left inguinal region, a symptom much complained of throughout the disease, persisted, resisting all treatment by medicine internally and externally, leeching, blistering, anodyne plasters, liniments, etc., for some months. I then, with a camel'shair pencil, well saturated with vesicating collodion, brushed over the cervix uteri briskly for a minute or so, which caused great stinging, pricking, and burning pain through the pelvis, lasting for some hours; when a watery discharge of a hot, scalding nature, and starching the linen, set in and remained for one day, being then succeeded by one of a purulent character, but not causing pain or soreness in the parts. As the pain still remained, but less intensely, the blistered surface was kept open for a fortnight by powdered nitrate of silver, when it was permitted to heal up, the inguinal pain having been much lessened; but as, at the end of another week, the pain was still persisting, I had recourse to the vesicating gutta percha No. 2, which did not cause pain, either during its application or otherwise. It produced the same phenomena as the collodion had done, but neither so quickly nor so actively. On the third day from its use, the pain which had given her so much annoyance had completely disappeared, and she has enjoyed better health ever since than she has had for many years before she submitted to treatment.

"CASE II. Mrs. C., aged 47, of a very bilious temperament, married upwards of two years, but never pregnant, was under treatment for acute inflammation of the cervix uteri and vagina, supposed to be of a gonorrhœal character. The disease was removed by warm baths, local bleeding by the lancet, and nitrate of silver, the bowels being kept well open; still, a burning pain in the pelvis, sacrum, and down the thighs (at all times much complained of), persisted, in spite of all means em. ployed for its removal. The vesicating collodion, and vesicating gutta percha No. 2, were used as in Case I; but there was not any relief until after two applications (at the interval of a week) of the vesicating gutta percha No. 1 , the effect of which was kept up for about ten days by the preparation No. 2 , at the end of which time she was quite well. Whilst the solution No. 1 was being used in this case, a slight pricking and stinging warmth was experienced, ceasing on the appearance of vesication, followed by a watery discharge which was evident before the speculum was withdrawn. On the third day after its use, the surface of the cervix was raw, like a blistered surface on other parts of the body; and the epithelium was in some parts thickened, whilst in others it was hanging detached, like chewed paper; a purulent discharge, mixed with water, then issued from the vagina, but produced no inconvenience.

"CASE III. Mrs. B., aged 21, of a nervous temperament, married about two years, but never pregnant, came under my care, being affected with chronic inflammatory engorgement of the cervix uteri, with retroversion of the organ, and a great amount of tenderness in the right ovary, which was found to be enlarged, as discoverable by the vagina and rectum. Under appropriate treatment, as bleeding internally by scarification, and externally by leeches, external blistering, mercury with sedatives, the mouth being gently touched, warm hip-baths, 